\title{
Article \\ A Novel Allele Encoding 7-Hydroxymethyl Chlorophyll a Reductase Confers Bacterial Blight Resistance in Rice
}

\author{
Marie Gorette Kampire ${ }^{1}$, Ringki Kuinamei Sanglou ${ }^{1}$, Huimei Wang ${ }^{1}$, Bello Babatunde Kazeem ${ }^{2}$, Jian-li Wu ${ }^{1, *(D)}$ \\ and Xiaobo Zhang $1, *$ (D) \\ 1 State Key Laboratory of Rice Biology, China National Rice Research Institute, Hangzhou 310006, China; \\ kamgorette@gmail.com (M.G.K.); ringki007@gmail.com (R.K.S.); wanghuimei@caas.cn (H.W.) \\ 2 Lianyungang Academy of Agricultural Sciences, Lianyungang 222006, China; tunlapa2k13@gmail.com \\ * Correspondence: beishangd@163.com (J.-1.W.); zhangxiaobo@caas.cn (X.Z.); Tel.: +86-571-63370326 (J.-1.W.); \\ $+86-571-63370295$ (X.Z.)
}

check for

updates

Citation: Kampire, M.G.; Sanglou, R.K.; Wang, H.; Kazeem, B.B.; Wu, J.-1.; Zhang, X. A Novel Allele Encoding 7-Hydroxymethyl Chlorophyll a Reductase Confers Bacterial Blight Resistance in Rice. Int. J. Mol. Sci. 2021, 22, 7585. https://doi.org/10.3390/ijms22147585

Academic Editor:

Pedro Martínez-Gómez

Received: 23 June 2021

Accepted: 13 July 2021

Published: 15 July 2021

Publisher's Note: MDPI stays neutral with regard to jurisdictional claims in published maps and institutional affiliations.

Copyright: (C) 2021 by the authors Licensee MDPI, Basel, Switzerland. This article is an open access article distributed under the terms and conditions of the Creative Commons Attribution (CC BY) license (https:// creativecommons.org/licenses/by/ $4.0 /)$.

\begin{abstract}
Rice spotted leaf mutants are helpful to investigate programmed cell death (PCD) and defense response pathways in plants. Using a map-based cloning strategy, we characterized novel rice spotted leaf mutation $\mathrm{spl}^{\mathrm{HM} 143}$ that encodes a 7-hydroxymethyl chlorophyll a reductase (OsHCAR). The wild-type (WT) allele could rescue the mutant phenotype, as evidenced by complementation analysis. OsHCAR was constitutively expressed at all rice tissues tested and its expression products localized to chloroplasts. The mutant exhibited PCD and leaf senescence with increased $\mathrm{H}_{2} \mathrm{O}_{2}$ (hydrogen peroxide) accumulation, increased of ROS (reactive oxygen species) scavenging enzymes activities and TUNEL (terminal deoxyribonucleotidyl transferase-mediated dUTP nick-end labeling) -positive nuclei, upregulation of PCD related genes, decreased chlorophyll (Chl) contents, downregulation of photosynthesis-related genes, and upregulation of senescence-associated genes. Besides, the mutant exhibited enhanced bacterial blight resistance with significant upregulation of defense response genes. Knockout lines of OsHCAR exhibited spotted leaf phenotype, cell death, leaf senescence, and showed increased resistance to the bacterial pathogen Xanthomonas oryzae pv. oryzae (Xoo) coupled with upregulation of five pathogenesis-related marker genes. The overexpression of OsHCAR resulted in increased susceptibility to Xoo with decreased expression of pathogenesis-related marker genes. Altogether, our findings revealed that OsHCAR is involved in regulating cell death and defense response against bacterial blight pathogen in rice.
\end{abstract}

Keywords: rice; bacterial blight; defense response; OsHCAR

\section{Introduction}

Rice bacterial blight is a deadly bacterial disease which is among the most destructive for cultivated rice (Oryza sativa). Spotted leaf mutants of rice known to provide extensive resistance to different bacterial pathogens serve as a great material to elucidate disease resistance mechanisms in rice. Understanding the functions of disease-resistant genes is essential for unraveling the molecular mechanisms of plant capacity to withstand pathogenic bacteria and improve plant adaptability to stress through genetic programming [1]. In rice, spotted leaf mutants are known to develop necrotic lesions spontaneously without any environmental stress intervention. The developed necrotic lesions are mainly observed on the leaf blade or leaf sheath and are of different sizes $(2-10 \mu \mathrm{m})$ and multiple colors such as brown, reddish-brown, dark brown, orange, and white [2,3]. Spotted leaf mutants are also termed lesion mimic mutants (LMMs) because they display either structural or uncontrolled cell death initiation similar to the hypersensitive response (HR) induced by pathogen infection [4]. HR is associated with typical physiological processes that comprise structural signals such as the initiation of pathogenesis-related (PR) genes, the outburst of ROS, the aggregation of antimicrobial compounds, and the generation of free radicals [5-7]. 
Genetic studies have stated that spotted leaf mutants are commonly governed by single recessive genes [8,9], single semi-dominant nuclear genes [10] or single dominant genes [11]. In recent years, many spotted leaf mutant genes have been cloned. They encode several proteins with distinct functions and signaling pathways associated with defense response and HR cell death in rice [12]. These proteins include ATP-citrate lyases (ACL) [13], spastin protein [14], coproporphyrinogen III oxidase [15], mitogen-activated protein kinase kinase kinase (MAPKKK1) [16], eEF1A-like protein [7] tetratricopeptide repeats (TPRs)-containing protein [17], and AAA-type ATPase [18]. These findings specify that several proteins are important regulators of HR cell death and immunity response [13].

Most spotted leaf mutants show improved defenses against bacterial and fungal pathogens. For example, rice spl40 mutant displayed increased resistance to bacterial blight pathogen Xoo [19], whereas Spl12, spl13, spl14 and Spl15 show enhanced resistance to not only Magnaporthe oryzae but also X. campestris pv. oryzae [20], and spl33 exhibited improved resistance to Magnaporthe oryzae and Xoo [7]. These data imply that spotted leaf genes participate in the regulation of the plant defense response. Certain gene products such as phosphates, G-proteins, and protein kinase, other signal molecules such as lipid peroxides, ROS, salicylic acid (SA), and jasmonic acid (JA) are produced during the defense response. In fact, at the beginning of defense response, many biochemical pathways within the responding cells are immediately activated, followed by the induction of defense-related genes, including genes encoded by chitinase, phenylalanine ammonia-lyase (PAL), and, chalcone synthase (CHS) plus protectant genes including genes encoding glutathione Stransferase (GST), glutathione peroxidase (GP) and peroxidases for activating cell protection mechanisms [21]. During the last decade, several LMM have been isolated and cloned. It is now recognized that these mutants are valuable materials for studying various features of PCD and the disease resistance mechanisms of rice [4].

7-hydroxymethyl chlorophyll a reductase (HCAR) is an enzyme of Chl cycle that accelerates the modification of 7-hydroxymethyl Chl a (7-HMC a) to Chl a [22]. In rice and Arabidopsis, HCAR is considered a component of Chl catabolic enzyme (CCE). It interferes with RCCR, SGR, NOL, and NYC1 in yeast two-hybrid experiments, suggesting its prominent role in Chl degradation $[23,24]$. A recent study demonstrated that HCAR acts as one of the suppressing factors for $\mathrm{Chl} b$ turnover when $\mathrm{Chl} b$ is overproduced. Analysis performed on Chl-b-overproducing plants and the lines that overexpress HCAR showed that the $\mathrm{Chl} b$ level was decreased. In contrast, the $\mathrm{Chl} \mathrm{a} / \mathrm{b}$ ratio was elevated from about 0.8-1 to 1.6-2, implying that HCAR activity was not enough to convert HMChl a reduction in Chl-b-overproducing plants [25]. In rice, HCAR has been reported to play a crucial role in saving plants from high light-induced cell injury by blocking the aggregation of 7- HMC a and Pheo a in evolving and mature leaves during plant development [24]. However, an association between disease resistance and HCAR has not been reported in plants so far.

Previously, a spotted leaf mutant obtained by the EMS (Ethyl methane sulfonate)induced IR64 mutant bank (HM143) showed necrotic lesions on the leaf blades and exhibited broad spectrum resistance to different bacterial blight pathogens [9]. To confirm whether the mutation in HM143 was associated with the spotted leaf phenotype and bacterial resistance we carried out functional analysis of this gene which would deepen our understanding of the molecular mechanisms underlying disease resistance in rice. Here, we isolated $\mathrm{spl}^{\mathrm{HM} 143}$, which encodes 7-hydroxymethyl chlorophyll a reductase, hereafter (OsHCAR). A single base exchange in the mutant allele leading to an mRNA splicing is responsible for the mutant phenotype indicated by complementation test. The OsHCAR gene is constitutively expressed in all tissues examined and OsHCAR localizes to chloroplasts. The mutant displayed cell death, leaf senescence and increased defense response. The knockout lines of OsHCAR exhibited similar spotted leaf phenotype, leaf senescence, cell death and strengthened disease resistance. Our results demonstrated that dysfunction of OsHCAR could activate the defense pathway and lead to enhanced defense response in rice. 


\section{Results}

\subsection{PCD Is Activated in HM143}

A previous study denoted that cell death occurred at every site of necrotic lesions, followed by the cumulation of hydrogen peroxide in HM143 [9]. To further confirm the presence of cell death in HM143, we performed TUNEL analysis to detect DNA fragmentation, which indicates cell death. We observed few positive TUNEL nuclei in the WT, whereas many TUNEL nuclei were positive in HM143 (Figure 1A). We also measured the levels of malonaldehyde (MDA) and $\mathrm{H}_{2} \mathrm{O}_{2}$. Our results revealed that the contents of both MDA and $\mathrm{H}_{2} \mathrm{O}_{2}$ were significantly increased in $H M 143$ compared with WT (Figure 1B,C), indicating that cell membrane damage caused cell death in HM143.
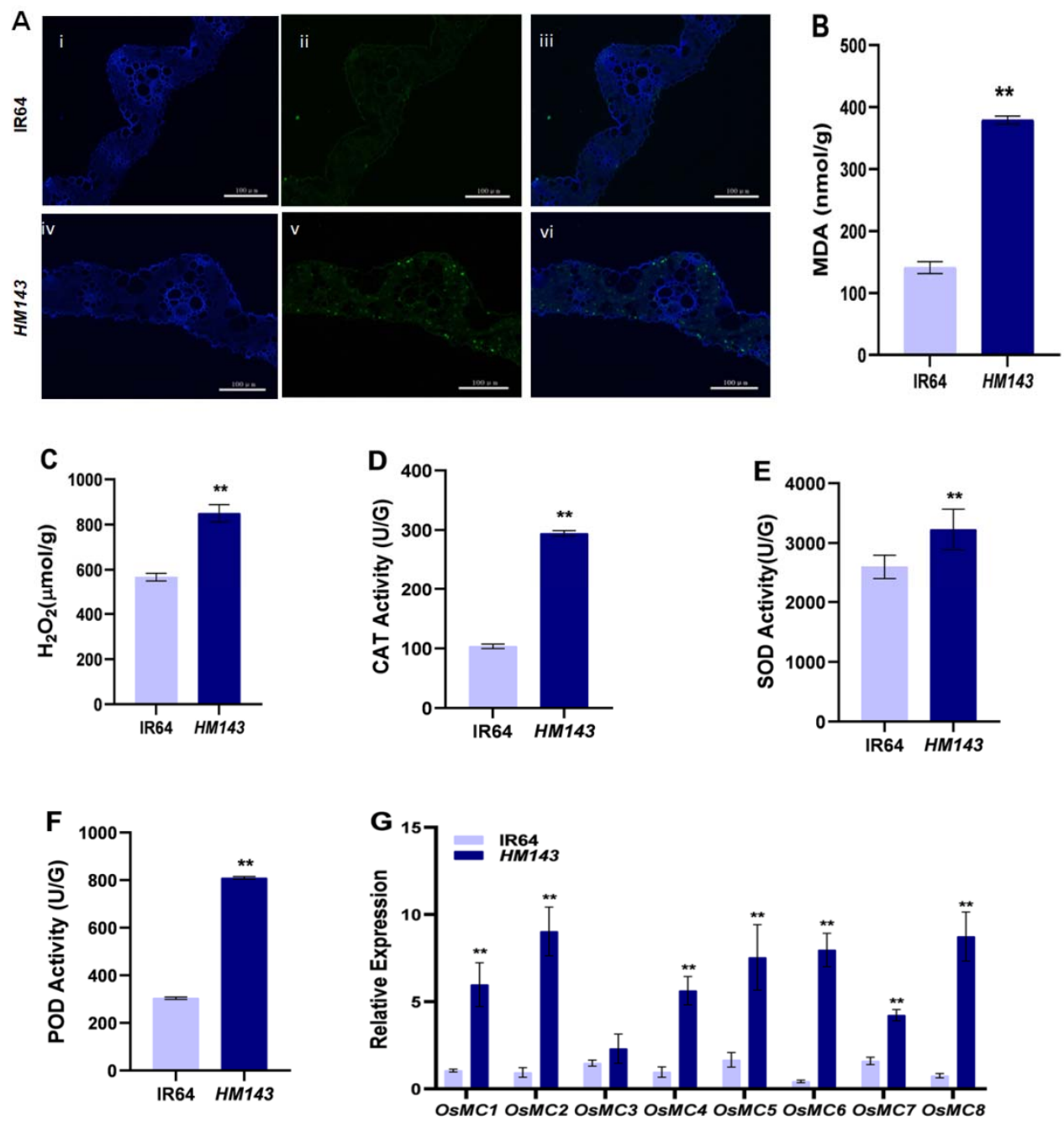

Figure 1. Analysis of cell death and ROS accumulation in IR64 and HM143 at tillering stage. (A) TUNEL assay. Blue signal represents 4' , 6-diamino-phenylindole (DAPI) staining; green color represents positive result. (i) and (iv) are DAPI staining; (ii) and (v) are TUNEL signal; (iii) and (vi) are merged images of (i/iv) andrespectively, $\mathrm{Bar}=100 \mu \mathrm{m}$; (B) Malonaldehyde (MDA) contents; (C) $\mathrm{H}_{2} \mathrm{O}_{2}$ contents; (D-F) CAT, SOD and POD enzyme activities (G) Expression analysis of OsMCs genes. Data are means \pm SD of three biological replicates (Student's $t$-test: ${ }^{* *} p<0.01$ ).

To verify whether anti-oxidative systems, consisting of catalase (CAT), superoxide dismutase (SOD), and peroxidase (POD) enzymes, were elevated to balance ROS production, we determined the activities SOD, POD and CAT between mutant and WT. We found out that the all the three enzyme activities were significantly higher in HM143 when compared with WT (Figure 1D-F). Our results imply that increased activities of ROS scavenging 
enzymes could not lower the elevated $\mathrm{H}_{2} \mathrm{O}_{2}$ accumulation. Lastly, we performed the expression analysis of several metacaspase (MC) genes, which are essential controllers of PCD [26]. The results revealed that seven MC genes, including OsMC1, OsMC2, OsMC4, OsMC5, OsMC6, OsMC7, and OsMC8, were highly expressed in HM143 compared to $\mathrm{WT}$, but no remarkable difference was detected in the expression pattern of $1 \mathrm{MC}$ gene, i.e., OsMC3 between HM143 and WT (Figure 1G). Our results demonstrated that HM143 triggers the PCD pathway, which led to the formation of HR-like necrotic lesions.

\subsection{HM143 Is Deficient in Chlorophyll Metabolism and Photosynthesis}

Photosynthetic rate (Pn) and $\mathrm{Chl}$ content are the two significant characteristics of leaf senescence in plants [27]. To examine early leaf senescence, we measured the levels of Chl contents in mutant and WT plants at the tillering stage (Figure 2A). We found that in HM143, Chl a, Chl b, and carotenoid (Cart) levels were notably lower than WT (Figure 2C), suggesting that necrotic lesions' formation in HM143 might have caused the reduction of $\mathrm{Chl}$ contents. We also observed that the net photosynthetic rate was significantly decreased in the HM143 plants as opposed to WT (Figure 2B). We also evaluated the expression levels of eight photosynthesis-related marker genes ( $r b c L, C H L D$, cab2R, CHLH, psbA, CHLI, porA, $H E M A 1, r b c S$ ) by qRT-PCR (Quantitative Real-Time Polymerase Chain Reaction) in the mutant and WT. All the photosynthesis-related genes tested were remarkably downregulated in the mutant compared to the WT (Figure 2D), providing a shred of molecular evidence for leaf senescence in the mutant. Our results imply that HM143 is deficient in Chlmetabolism and photosynthesis.

A
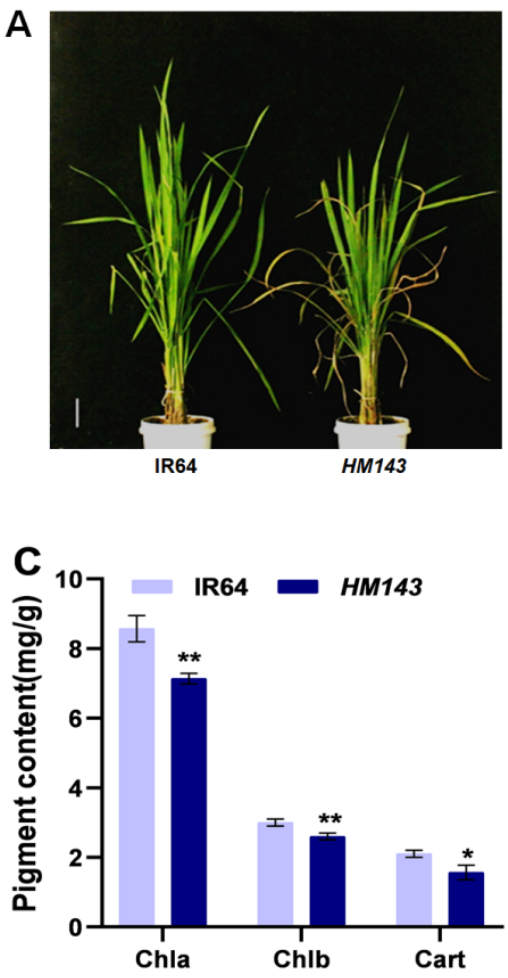
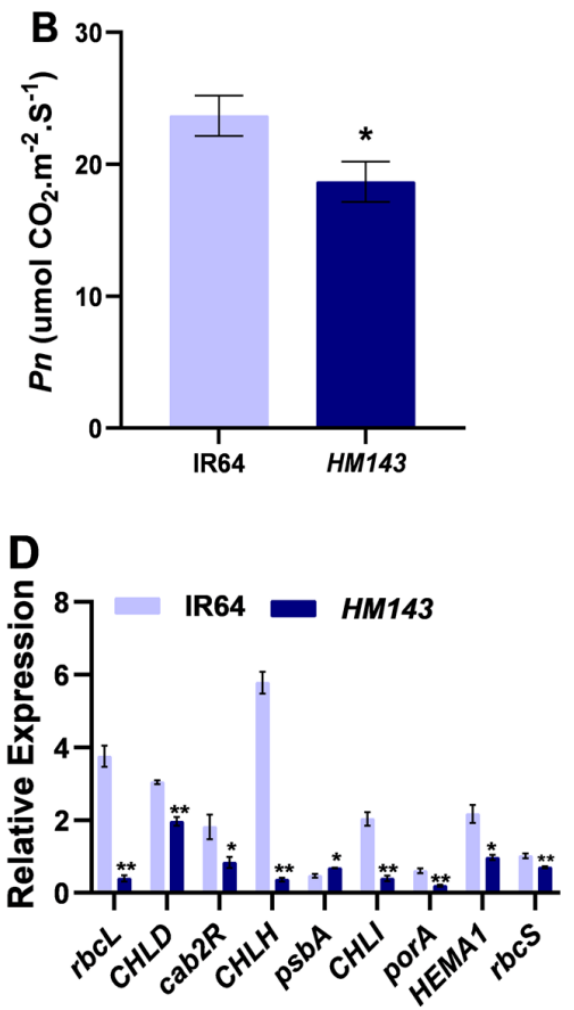

Figure 2. Analysis of leaf senescence in IR64 and HM143 at the tillering stage. (A) Phenotypes of IR64 and HM143 at the tillering stage, bar $=17 \mathrm{~cm} ;(\mathbf{B})$ Net Photosynthetic rate $(\mathrm{Pn}) ;(\mathbf{C})$ Pigment content of IR46 and HM143; (D) Expression levels of photosynthetic related genes. Data are means \pm SD of three biological replicates (Student's $t$-test: ${ }^{*} p<0.05$; ${ }^{* *} p<0.01$ ).

\section{3. spl ${ }^{H M 143}$ Encodes 7-Hydroxymethyl Chlorophyll a Reductase (OsHCAR)}

In a previous study, the single recessive gene $s p l^{H M 143}$ was preliminarily mapped to chromosome 4 [9]. A sum of $1380 \mathrm{~F}_{2}$ individuals mutant-type obtained from the cross 
HM143/Moroberekan was selected for genotyping to fine map the locus. The candidate gene was located to a $176 \mathrm{~Kb}$ region between RM16682 and RM16686 markers (Figure 3A). Elicited from the Rice Genome Annotation Project database (http:/ / rice.plantbiology.msu. edu/, accessed on 23 June 2021), the fine mapped region contains 20 putative open reading frames (ORFs) (Figure 3B). Sequencing and comparing all 20 ORFs from WT and HM143 indicated that the 14th ORF (LOC_Os04g25400) had one nucleotide exchange from T to A at position 3760 in its 12th intron splicing site (Figure 3D) resulting in mRNA splicing, thus considering LOC_Os04g25400 as the most likely candidate gene. The length of LOC_Os04g25400 is $5273 \mathrm{bp}$ with 16 exons and 16 introns (Figure 3C). LOC_Os04g25400 is predicted to encode 7-hydroxymethyl chlorophyll a reductase (HCAR), hence named $\mathrm{OsH}^{-}$ CAR.

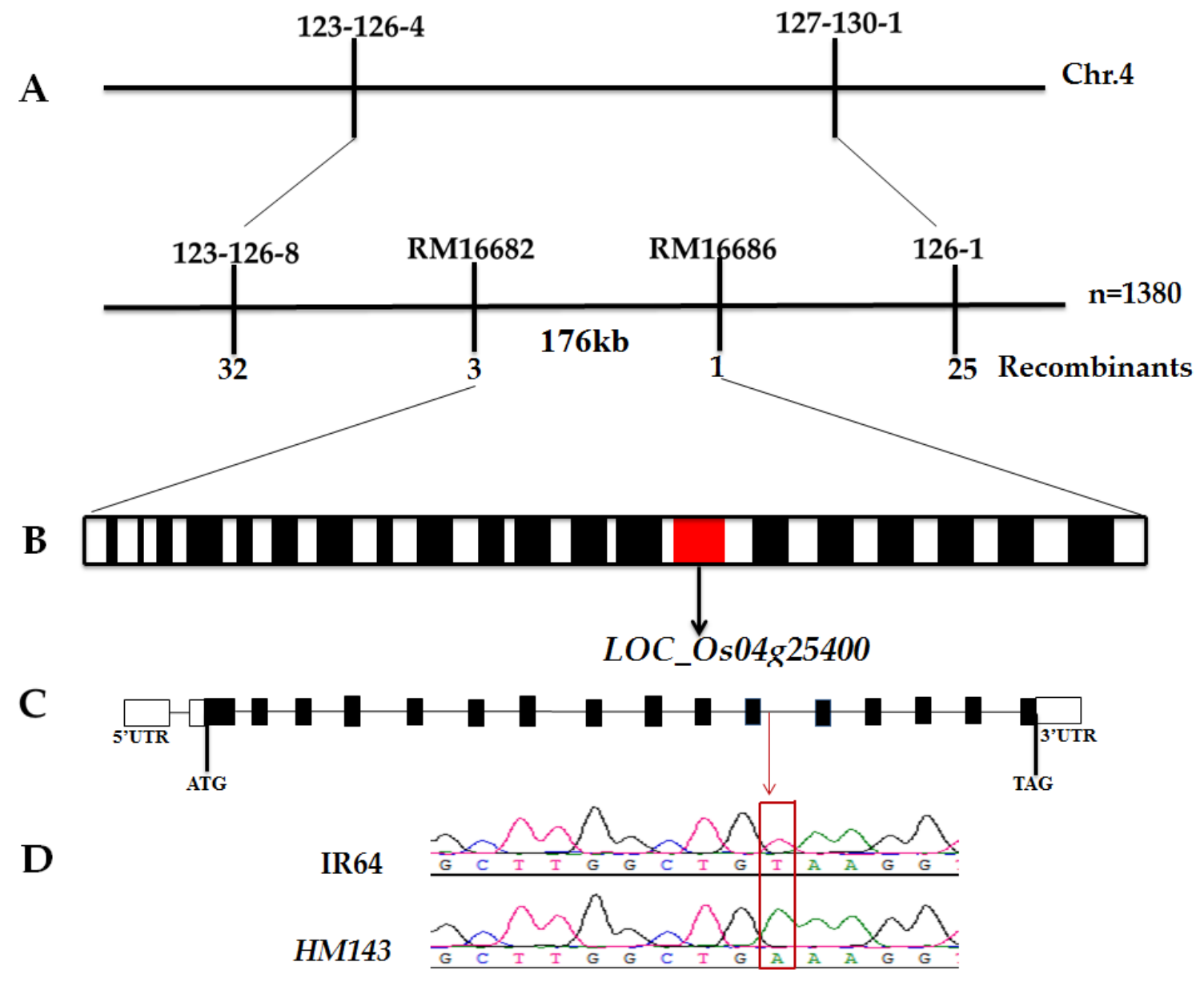

Figure 3. Map based cloning of OsHCAR. (A) OsHCAR gene location on chromosome 4 between markers RM16682 and RM16886. (B) 20 ORFs were located in 176kb region, red box represents LOC_Os04g25400, and black boxes represent other ORFs. (C) Gene structure of LOC_Os04g25400, white boxes represent 5'UTR and 3'UTR, respectively, while black boxes represent coding exons, lines represent introns. (D) Sequence analysis of the T to A point mutation in WT and HM143 on the second nucleotide of $12^{\text {th }}$ intron. Note: The numbers $123-126-4,127-130-1,123-126-8,126-1$ indicate insertion/deletion markers.

To confirm the function of the candidate gene, we performed Agrobacterium tumefaciensmediated transformation of the wild OsHCAR allele in the mutant calli (Figure 4A). Eight $\mathrm{T}_{0}$ plants were regenerated; six were positive transformants and displayed the same regular green leaf color as the WT(Figure 4B); while the other two showed the same lesion mimic phenotype as the mutant (Supplementary Figure S1A,B). We also measured the total Chl content in IR64, HM143, and C-HM143. Our results showed that the total Chl level in CHM143 recovered to the WT levels while it significantly lowered in HM143 (Supplementary 
Figure S1C). Additionally, when we measured the content of Chl, the mutant showed decreased levels of $\mathrm{Chl} a, b$, and Cart compared with WT: these parameters rescued WT levels in complementary plants (Figure $4 \mathrm{C}$ ). Furthermore, we also measured the enzyme activities of POD, SOD and, CAT and our results showed that POD and SOD activities in the complementary plants fully recovered to the WT level (Figure $4 \mathrm{D}, \mathrm{F}$ ). In contrast, the activity of CAT was decreased compared to WT but higher than that of HM143 (Figure 4E). Joined together, these findings illustrated that LOC_Os04g25400 is the candidate gene that regulates the mutant phenotype.

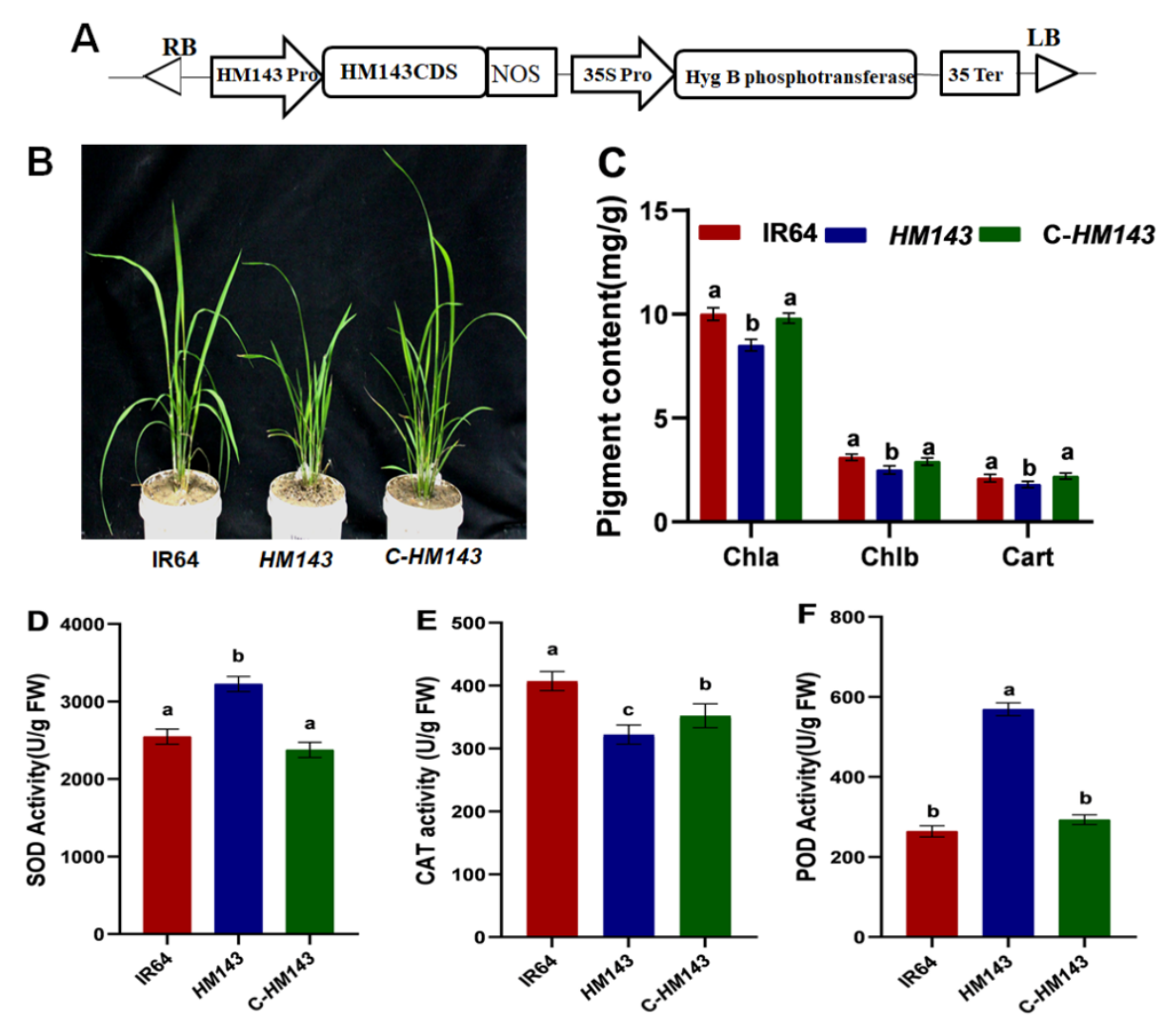

Figure 4. Genetic complementation of the mutant. (A) Complementation construct. (B) Phenotypes of IR64, HM143 and complemented transgenic line (C-HM134). (C) Pigment contents in IR64, HM134 and C-HM143 at heading stage. (D-F) Enzyme activities of SOD, CAT and POD in IR64, HM143 and C-HM143 at heading stage. Data are means \pm SD of three biological replicates. Different letters indicate significant differences according to One-way ANOVA and Duncan's test $(p \leq 0.05)$.

\subsection{Overexpression of OsHCAR Enhances Susceptibility to Xoo}

To evaluate the biological function of OsHCAR, we produced transgenic rice overexpressing the WT OsHCAR allele under the control maize (Zea mays) Ubiquitin1 in the Kitaake background. A total of four OsHCAR $\mathrm{T}_{0}$ overexpression plants (OE) were obtained, and they displayed no apparent morphological changes compared to Kitaake (Figure 5A). The expression levels of OsHCAR in the $\mathrm{T}_{0}$ OE plants were significantly increased compared to Kitaake (Figure 5B). For further analysis, T1 OE lines were planted in the field, and we measured the activities of SOD and CAT, which are increased during oxidative stress. In SOD activity, the results showed no significant difference between Kitaake and OE lines (Figure 5C). In contrast, CAT activity was significantly higher in OE lines than Kitaake (Figure 5D). 

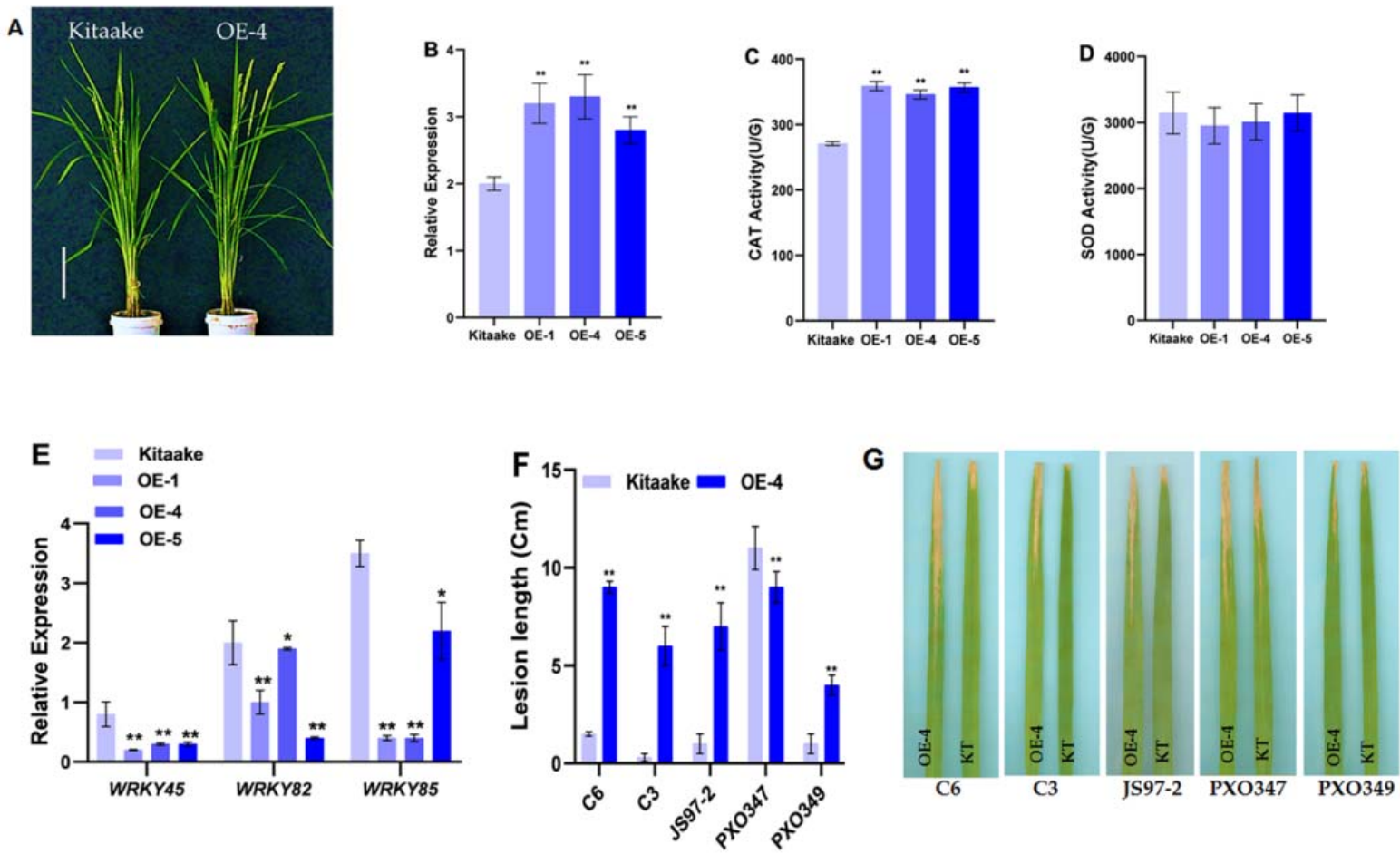

Figure 5. Overexpression analysis of OsHCAR in Kitaake background. (A) Phenotypes of Kitaake and $\mathrm{T}_{1}$ Overexpression line (OE-4). (B) Relative expression of OsHCAR in Kitaake and different OE lines. (C,D) Enzyme activities of CAT and SOD (E) Expression levels of Pathogenesis related genes. (F) Lesion length (cm). (G) Leaf phenotypes of Kitaake and OE-4 plants after inoculation with bacterial blight pathogens. Data are means \pm SD of three biological replicates (B-E) and five biological replicates (F) (Student's $t$-test: ${ }^{*} p<0.05 ;{ }^{* *} p<0.01$ ).

It has been reported that HM143 enhances disease resistance to multiple bacterial blight pathogens [9]. To access the disease response to bacterial blight pathogens in the $\mathrm{OE}$ lines, we performed bacterial inoculation of five different bacterial blight races, namely C3, C6, JS97-2, PXO347, and PXO349, between the Kitaake and OE-4 lines. We found that the OE-4 lines were more susceptible to bacterial blight pathogens compared to Kitaake, except for PXO347 (Figure $5 \mathrm{~F}, \mathrm{G}$ ). We examined the expression pattern of three different pathogenesis-related (PR) genes (WRKY45, WRKY82, and WRKY85). The results showed that these PR genes were poorly expressed in the OE-4 plants compare with Kitaake (Figure 5E). These findings demonstrate that the OsHCAR gene is involved in regulating bacterial blight resistance and that overexpression of OsHCAR enhances disease susceptibility in rice.

\subsection{Knockout of OsHCAR Promotes Resistance to Xoo}

We used thef CRISPR/Cas9 technique to generate knockout lines of OsHCAR using the WT as the background. The sequenced CRISPR/Cas9 target sites revealed several insertions in the cr-12, cr-18, and deletion in the cr-19 (Figure 6A). The three indicative lines were used for comprehensive characterization. To verify the off-target effect, we obtained 20 off-target sites from the CRISPR-P website (http:/ / cbi.hzau.edu.cn/cgi-bin/CRISPR, accessed on 23 June 2021) (Supplementary Table S2), and we detected those off sequence in cr-12, cr-18 and cr-19 by PCR (Polymerase Chain Reaction) amplification and sequencing to verify whether they resulted in any insertion, deletion or substitution mutation. We did not detect any change in cr-12, cr-18, and cr-19 regarding the 20 off-target sites (Supplementary Figures S3-S7), and thus concluded that our guide RNA sequence was only specific to the OsHCAR gene. 

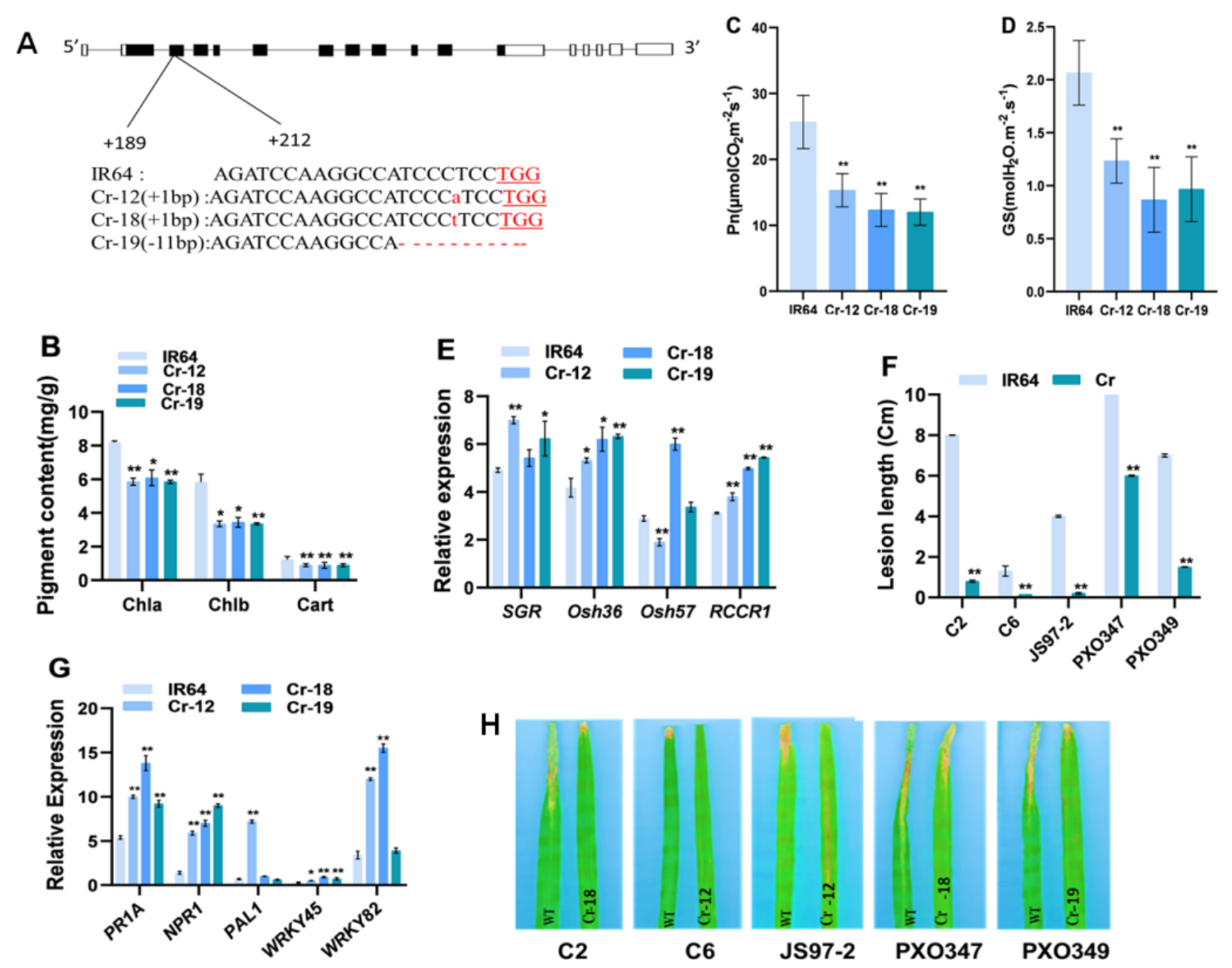

Figure 6. Analysis of OsHCAR knockout lines using CRISPR/Cas9. (A) CRISPR/CAS9 mediated mutation at target sites of OsHCAR in knockout lines. $\mathrm{Cr}-12, \mathrm{Cr}-18$ are homozygous mutants carrying 1 bp insertion whereas $\mathrm{Cr}-19$ carries $11 \mathrm{bp}$ deletion, the black letters represent the sgRNA target sequence and the underlined red letters represents PAM motif. (B) Chlorophyll content, (C) Net photosynthetic rate (Pn). (D) Stomatal conductance (Gs). (E) Relative expression of senescence genes. (F) Lesion length (cm). (G) Relative expression analysis of pathogenesis related genes in WT and knockout lines and $(\mathbf{H})$ leaf phenotypes of WT and knockout lines after bacterial blight inoculation. Data are means \pm SD of three biological replicates (B-E,G) and five biological replicates (F) (Student's $t$-test: ${ }^{*} p<0.05 ;{ }^{* *} p<0.01$ ).

As expected, the knockout lines exhibited a spotted leaf phenotype similar to the mutant with decreased levels of $\mathrm{Chl}$ a, b, and Cart (Figure 6B) and increased expression levels of senescence-associated genes compared to WT (Figure 6E). We speculated that the reduction in the $\mathrm{Chl}$ content in the knockout lines might cause alterations in photosynthetic parameters, so we quantified the photosynthesis parameters. We observed a significant reduction in the $P n$ and stomatal conductance (GS) in the knockout lines compared to WT (Figure 6C,D), indicating that the knockout lines had defective photosynthetic capacity, which possibly led to reductions in Chl content and lesion formation.

To analyze the disease resistance, knockout lines and WT plants were inoculated with five races of Xoo, i.e., C2, C6, JS97-2, PXO347, and PXO349, and the lesion length was measured two weeks after inoculation. The knockout plants showed enhanced disease resistance to each of the five Xoo races compared with WT (Figure 6F,H). PR genes are frequently enhanced during lesion formation in many rice spotted-leaf mutants. During pathogen infection, improved disease resistance is accompanied by the elevation of PR genes [28]. To verify this possibility, we performed an expression analysis of five defenserelated marker genes (PR1A, NPR1, PAL1, WRKY45, WRKY82) by qRT-PCR. We found that each of these PR marker genes was significantly increased in the knockout plants compared 
to the WT (Figure 6G). Overall, these results indicate that the dysfunction of OsHCAR results in cell death and activates disease resistance in rice.

\subsection{Constitutively Expressed OsHCAR Is Localized to Chloroplasts}

To examine the subcellular localization of OsHCAR experimentally, we performed polyethylene glycol (PEG)-mediated rice transformation in rice protoplasts. When the pOsHCAR-GFP vector was transformed in rice protoplasts controlled by CaMV 35S promoter, the green fluorescence signals of OsHCAR: GFP were confined to the chloroplasts (Figure 7A). Our results demonstrated that OsHCAR is a chloroplast-targeted protein.
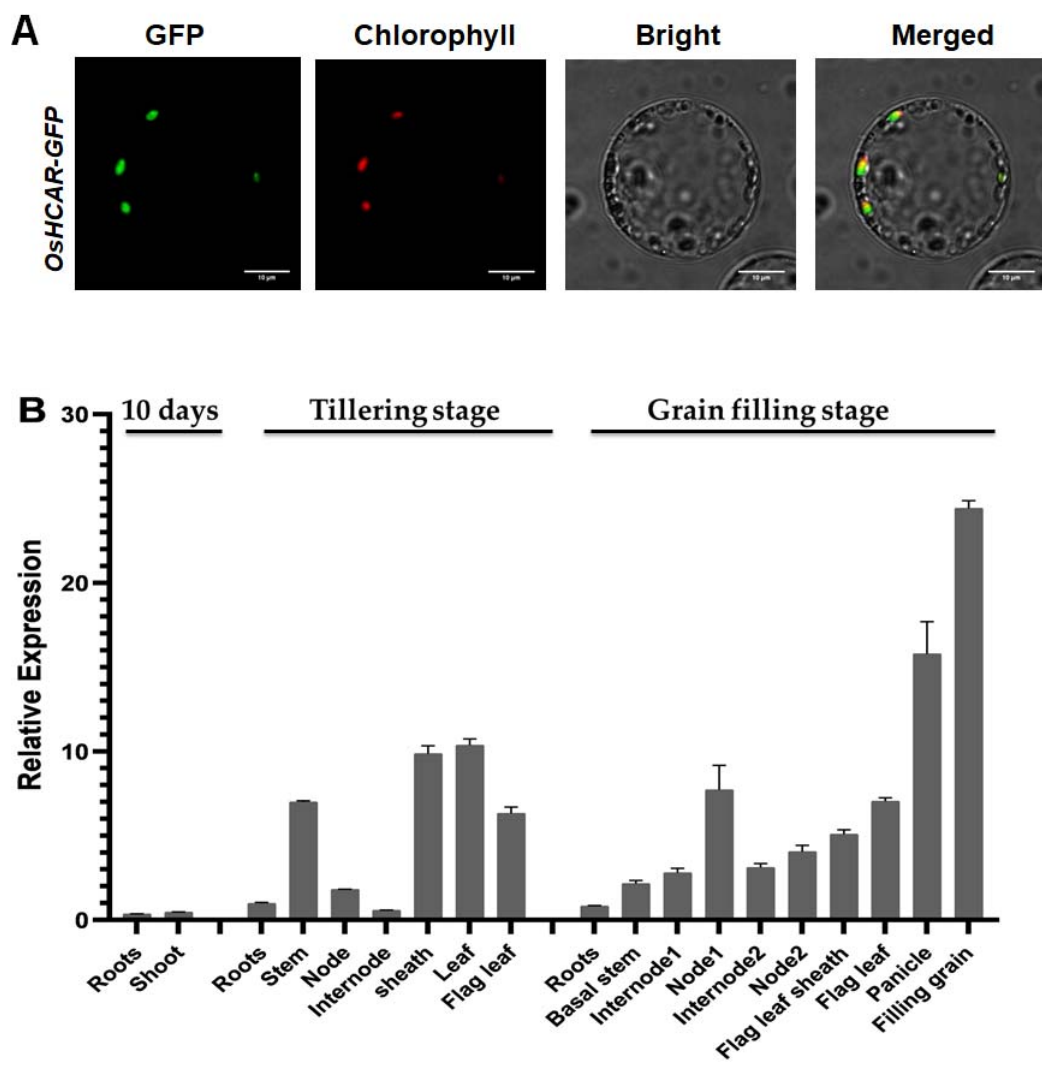

Figure 7. qRT-PCR analysis of wild-type OsHCAR messenger RNA expression and subcellular localization of OsHCAR. (A) Subcellular localization of OsHCAR-GFP in rice protoplasts. Bar $=10 \mu \mathrm{m}$ (B) Quantitative relative expression levels of OsHCAR in various tissues of IR64 at different growth stages. Rice ubiquitin gene (LOC_Os03g13170) was used as reference gene.

To verify the expression system of OsHCAR, we evaluated the expression levels of total RNA from roots, shoots, stems, nodes, internodes, leaves, leaf sheaths, panicles, and filling grains by quantitative reverse transcription-polymerase chain reaction at different developmental stages. Our results indicated that the OsHCAR gene was highly expressed in all the tissue evaluated, with the highest expression in the filling grains (Figure 7B). These findings reveal that OsHCAR is a constitutively expressed gene.

\section{Discussion}

Spotted leaf mutants are essential for comprehending PCD and disease resistance in plants. Previously, we isolated a spotted leaf mutant (HM143) from an IR64 treated EMS mutant bank. The target gene was originally termed $s p l^{H M 143} . s p l^{H M 143}$ exhibited PCD and improved disease resistance to several races of Xoo [9]. In the present study, the mutant allele splHM143, which possesses one nucleotide replacement at the splicing site, was identified as LOC_Os04g25400 after fine mapping; and it encodes for a rice 7hydroxymethyl chlorophyll a reductase (OsHCAR) protein. Complementation analysis 
using WT LOC_Os04g25400 could rescue the spotted leaf phenotype. HCAR catalyzes the conversion of 7-hydroxymethyl Chlorophyll a (7-HMC a) to Chl a; and rice (Oryza sativa) genome comprises of one HCAR homolog [24]. OsHCAR is highly constitutively expressed and localizes to chloroplasts.

Mutation in many spotted leaf mutants is characterized by the poor performance of essential agronomic traits and decrease in photosynthetic pigments [29]. Still, our results revealed that there was a decrease in photosynthetic pigment. On the contrary, the performance of essential agronomic traits was substantially similar in HM143 mutant and the knockout lines than WT, suggesting that OsHACR may have a potential function in yield improvement due to enhanced disease resistance. HM143 exhibited a spontaneous leaf lesions resembling HR that occurs after pathogen infection. Previous studies have demonstrated that rice and Arabidopsis HCAR proteins regulate cell death and oxidative stress response. The intensity levels of $\mathrm{H}_{2} \mathrm{O}_{2}$ and $\mathrm{O}^{2-}$ were much higher in oshcar mutant leaves indicated by DAB and NBT staining. Additionally, JAmyb, OsNAC4, and OsAPX1 genes that are enhanced during cell death were notably upregulated, suggesting that HCAR plays a vital role in modulating cell death [24]. In this study, cell death in $\mathrm{OsH}_{-}$ $C A R$ was detected using TUNEL assay, an indicator of DNA fragmentation, which was reinforced by the upregulation of OsMCs genes which are essential regulators of PCD in plants [26]. $\mathrm{H}_{2} \mathrm{O}_{2}$ is a significant by-product of beta-oxidation and functions as a cue particle to promote cell death. Thus, over-accumulation of $\mathrm{H}_{2} \mathrm{O}_{2}$ is the leading cause of lesion development [30]. Accumulation of MDA causes cellular membrane damage and indirectly affects cell death [31]. Similarly, there was an elevated level of $\mathrm{H}_{2} \mathrm{O}_{2}$ and MDA in HM143, indicating an accumulation of cell death which might have contributed to the formation of necrotic lesions. Promoted level of ROS leads to oxidative damage and activation PCD pathway. If ROS appears as a damaging or signaling molecule is determined by the balance between ROS production and scavenging. Detoxing or scavenging of ROS is attained by an effective antioxidative system consisting of enzymatic antioxidants such as SOD, POD, and CAT to balance the production and removal of ROS [32]. Interestingly, CAT, SOD, and POD activities increased significantly in HM143; however, the level of ROS accumulation remained high. Further evaluation is needed to elucidate the issue.

In rice and Arabidopsis, HCAR participates in preventing cell death signaling during leaf senescence [23]. In general, leaf senescence is unified with reduction of Chl contents, defective chloroplast development, altered expression of photosynthesis-related genes, and elevated levels of Chl biosynthesis genes $[27,33,34]$. Similarly, in our study, we detected early leaf senescence. Our results revealed a reduction in the content of $\mathrm{Chl}$ and upregulation of $\mathrm{Chl}$ biosynthesis genes, and lowered expression of photosynthesis-related genes in HM143. Besides, we demonstrated decreased Chl content and upregulation of Chl biosynthesis genes in OsHCAR-knockout lines, indicating its function in leaf senescence. All the results obtained provided evidence that HCAR-like protein is involved in leaf senescence. It is believed that plant defense responses are regulated by endogenous plant signaling particles, including SA, JA, ethylene, and abscisic acid. For example, the bacterial blight resistance in OsPELOTA has been activated by the salicylic acid metabolic pathway [35], and simultaneously, the enhanced resistance observed in rice spl40 was due to activation of the SA and JA signaling pathways [19]. The mediated disease resistance of OsSPL24 was incidentally related to numerous pathways/components involved in heat shock proteins, vesicle trafficking, transcription factors, and cell wall components [10]. Salicylic acid (SA) and jasmonic acid (JA) are two practical regulators of defense reaction in plants and are believed to trigger a standard pathogen defense response in rice [36]. This study revealed that expression levels of PR markers involved in SA/JA were obviously elevated in the mutant plants, indicating that this gene is involved in defense response (Supplementary Figure S2A,B). Furthermore, OsHCAR-knockout lines improved resistance to rice five races of Xoo by inoculation experiments. There was a remarkably increased level of six PR genes in knockdown lines compared to WT. On the contrary, OsHCAR overexpressing plants showed susceptibility to five races of Xoo tested. Furthermore, the 
expression levels of three different PR markers were also decreased in the overexpression plants, suggesting that overexpression of OsHCAR may lead to the loss of function protein in regulating disease resistance. Previous reports demonstrated the importance of HCAR in Chl disintegration and cell death during leaf senescence [22,24]. This study demonstrated the potential role of OsHCAR in controlling bacterial blight pathogens in rice. Currently, transcriptome analysis is being conducted to uncover the molecular mechanism linking the Chlmetabolism pathway and disease resistance.

No previous reports have suggested the essential role of HCAR in defense response in rice. Overall, our results indicated that splHM143 encoded by HCAR has a significant role in promoting cell death and defense response in rice.

\section{Materials and Methods}

\subsection{Plant Materials and Growth Conditions}

The WT IR64 and HM143 mutant obtained from an EMS-induced mutant bank of IR64 were used in this study. During the summer of 2020, the IR64 and HM143 plants were grown in the paddy field, whereas transgenic rice, including overexpression plants, knockout plants, and complementation plants, were maintained in the greenhouse at the China National Rice Research Institute (CNRRI) in Fuyang, Hangzhou, China during different seasons of the years 2019 and 2020.

\subsection{Physico-Biochemical Parameters Measurement}

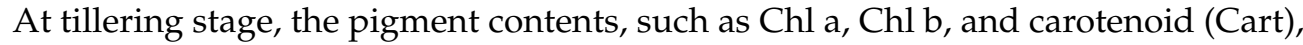
were evaluated in the top second leaves of HM143 and IR64 according to the method described by [37]. Similarly, the enzymatic activities of CAT, POD, and (SOD; and the levels of $\mathrm{H}_{2} \mathrm{O}_{2}$ andMDA were determined by employing the respective assay Kit of (Nanjing Jiancheng Bioengineering Institute, Nanjing, China) following the manufacturer's instructions. The mean value of three biological replicates was employed for analysis by Student's $t$ test, one-way ANOVA and Duncan's test.

\subsection{TUNEL Assay}

Samples of HM143 and IR64 were selected at the tillering stage for TUNEL assay following the manufacturer's requirements of the Fluorescein In Situ Cell Detection kit (Roche, Basel, Switzerland). Fluorescence labelling and sectioning were conducted as previously discussed by [38]. Leaf samples were visualized by viewing with laser scanning confocal microscope (Ceise, Jena, Germany).

\subsection{Vector Construction}

For complementation test, full-length CDS sequence (1800 bp) and promoter sequence (2000 bp) from IR64 were, respectively, amplified and inserted between HindIII and KpnI sites of pCAMBIA1300 vector. The recombinant vector was transformed into embryogenic calli obtained from HM143 via agrobacterium-mediated transformation. For overexpression construct, the total length of CDS $1800 \mathrm{bp}$ was amplified from IR64 by PCR and then cloned into pCAMBIA1300 vector powered by maize (Zea mays) Ubiquitin1 promoter. The recombinant vector was transformed into the mature calli generated from Kitaake via Agrobacterium tumefaciens-mediated modification. The CRISPR/Cas9 construct for OsHCAR was generated according to a previous report [39] and transformed into the calli generated from WT via Agrobacterium tumefaciens-mediated modification. All genetic transformations via Agrobacterium tumefaciens were performed using the method described by [40].

\subsection{Quantitative RT-PCR}

The total RNA from differing rice tissue, including root, shoot, internode1, node1, internode2, node2, flag leaf sheath, flag leaf, panicle, and filling grain was extracted following to the manufacturer's instructions using NucleoZOL Reagent Kit (MACHERYNAGEL, Düren, Germany). Briefly, $0.1 \mathrm{~g}$ tissue was homogenized with $1 \mathrm{~mL}$ Nucleozol 
reagent, then $400 \mu \mathrm{L}$ DEPC (Diethyl Polycarbonate) water was added to the lysate and mixed vigorously for $15 \mathrm{~s}$ and incubated for 5-15 $\mathrm{min}$ at room temperature. The mixture was centrifuged at 12,000 rpm for $15 \mathrm{~min}$. The supernatant was transferred to a new test tube and $100 \mathrm{~mL}$ of $100 \%$ isopropanol was added and incubated for $10 \mathrm{~min}$ at room temperature. After centrifugation for $10 \mathrm{~min}$ at 12,000 rpm, the supernatant was discarded and the pellet was washed twice by adding $500 \mu \mathrm{L}$ of $75 \%$ ethanol and centrifuging for $3 \mathrm{~min}$ at $8000 \mathrm{rpm}$ and the total RNA was reconstituted in $20 \mathrm{~mL}$ DEPC water. The cDNA was synthesized using the PrimeScript ${ }^{\mathrm{TM}}$ RT Master Mix (Perfect Real Time) (Takara, Dalian, China). For the qRT-PCR assay, PowerUp ${ }^{\mathrm{TM}} \mathrm{SYBR}^{\mathrm{TM}}$ Green master Mix kit was employed and conducted on a Thermal Cycler Dice ${ }^{\circledR}$ Real-Time System (Takara, Dalian, China) according to the following steps: Hold: $95^{\circ} \mathrm{C} 30 \mathrm{~s}$ (one cycle); two steps PCR: $95^{\circ} \mathrm{C}$ $5 \mathrm{~s}, 60{ }^{\circ} \mathrm{C} 30 \mathrm{~s}$ ( 40 cycles); dissociation at $95^{\circ} \mathrm{C}$ for $15 \mathrm{~s}$ ( 40 cycles); $60{ }^{\circ} \mathrm{C}$ for $30 \mathrm{~s}$, and $95^{\circ} \mathrm{C}$ for $15 \mathrm{~s}$. For the reference gene rice ubiquitin (LOC_Os03g13170) was utilized. Three replicates were used for all assays and the means were employed for calculations.

\subsection{Disease Evaluation}

At tillering stage, five fully expanded leaves of the WT, knockout lines, Kitaake, and OE-4 plants were inoculated with several bacterial races (C2, C3, C6, JS97-2, PXO347, and PXO349) of Xoo at the tillering stage following the leaf-clipping method described by [41]. For inoculation, distilled water was used to suspend bacterial cultures and calibrated to $\mathrm{OD}_{600}=1.0$. Disease lesion range was calibrated 14 days after inoculation using a ruler and the leaves were photographed by HP Scanjet G4010 scanner machine (HP, Shanghai, China). The mean value of five independent leaves per each bacterial race was used for analysis by Student's $t$ test.

\subsection{Subcellular Localization}

To prepare rice protoplasts, $0.5 \mathrm{~g}$ of rice seedlings were sliced into $0.5 \mathrm{~mm}$ size and digested in $10 \mathrm{~mL}$ enzyme solution (0.6 M Mannitol, $10 \mathrm{mM}$ MES, $1 \%$ Cellulose R10, 0.5\% Maceroezyme R10, $0.1 \% \mathrm{BSA}, 1 \mathrm{mM} \mathrm{CaCl} 2 \mathrm{pH}=7.5$ ) for $6 \mathrm{~h}$ in darkness with gentle shaking $(60 \mathrm{rpm})$. The protoplasts were filtered with a nylon mesh and collected by centrifugation, then washed twice with $10 \mathrm{~mL}$ ice-cold W5 solution $(9 \% \mathrm{NaCl}, 125 \mathrm{mM} \mathrm{KCl}, 5 \mathrm{mM}$ Glucose, $5 \mathrm{mM}$ MES $\mathrm{pH}=5.70$ ) followed by MMG solution ( $0.4 \mathrm{M}$ mannitol, $15 \mathrm{mM} \mathrm{MgCl}_{2}, 4 \mathrm{mM}$ MES, $\mathrm{pH}=5.8$ ), respectively.

The entire coding sequence of OsHCAR without a stop codon was amplified from WT (IR64) using specific primers 143CDS-1 and 143CDS-2, respectively (Supplementary Table S1). The PCR product of OsHCAR was merged with GFP in PAN580 vector controlled by the CaMV $35 \mathrm{~S}$ promoter to generate pOsHCAR-GFP construct. The new construct was then co-transiently expressed into rice protoplasts obtained from IR64 seedlings. The GFP signal was detected by viewing using a Zeiss 1 sm710 confocal microscope (Carl Zeiss, Inc., Jena, Germany) $48 \mathrm{~h}$ after transformation.

\subsection{Map-Based Cloning}

Previously, the mutation was mapped to the chromosome 4 [9]. For fine mapping, a total of $1380 \mathrm{~F}_{2}$ individuals of mutant-type derived from the cross HM143/Moroberekan were used. Simple sequence repeat (SSR) markers were retrieved from the (http:/ / www. gramene.org/, accessed on 23 June 2021) database whereas insertion/deletion (InDel) markers were synthesized using primer 5.0 and DNAStar 8.0 software after sequence comparison between the Indica cultivar 9311 and japonica cultivar Nipponbare from the Gramene public database (http:/ / gramene.org/genome_browser/index.html, accessed on 23 June 2021). Sangon Biotech Co. Ltd. (Shanghai, China) was used to synthesize the primers. PCR reaction and detection were performed as reported before by [8]. The suitable primer sequences for fine mapping are given in Supplementary Table S1. 
Supplementary Materials: The following are available online at https: / www.mdpi.com/article/ 10.3390/ijms22147585/s1, Figure S1: Validation of HM143 by complementation test, Figure S2: Expression analysis of defense genes involved in SA and JA pathways. Table S1: List of primers used in this study, Table S2: List of 20 potential off-target sites, Figures S3-S7: Sequence alignments of off-target sites in three knockout lines (Cr-12, Cr-18, Cr-19).

Author Contributions: Data curation, M.G.K.; Formal analysis, M.G.K. and R.K.S.; Investigation, X.Z.; Methodology, H.W. and B.B.K.; Supervision, X.Z. and J.-1.W.; Validation, X.Z.; Visualization, J.-1.W.; Writing—original draft, M.G.K. and J.-1.W. All authors have read and agreed to the published version of the manuscript.

Funding: This research was funded by [Central Public-interest Scientific Institution Basal Research] grant number [2017RG002-2].

Institutional Review Board Statement: Not applicable.

Informed Consent Statement: Not applicable.

Data Availability Statement: Data is contained within the article or supplementary material.

Acknowledgments: This research was supported by the Central Public-interest Scientific Institution Basal Research Fund of China National Rice Research Institute (2017RG002-2).

Conflicts of Interest: The authors declare no conflict of interest.

\section{References}

1. Kumar, V.; Jain, P.; Venkadesan, S.; Karkute, S.G.; Bhati, J.; Abdin, M.Z.; Sevanthi, A.M.; Mishra, D.C.; Chaturvedi, K.K.; Rai, A.; et al. Understanding Rice-Magnaporthe Oryzae Interaction in Resistant and Susceptible Cultivars of Rice under Panicle Blast Infection Using a Time-Course Transcriptome Analysis. Genes 2021, 12, 301. [CrossRef] [PubMed]

2. Wu, C.; Bordeos, A.; Madamba, M.R.S.; Baraoidan, M.; Ramos, M.; Wang, G.L.; Leach, J.E.; Leung, H. Rice Lesion Mimic Mutants with Enhanced Resistance to Diseases. Mol. Genet. Genom. 2008, 279, 605-619. [CrossRef]

3. Huang, Q.N.; Yang, Y.; Shi, Y.F.; Chen, J.; Wu, J.L. Spotted-Leaf Mutants of Rice (Oryza Sativa). Rice Sci. 2010, 17, 247-256. [CrossRef]

4. Moeder, W.; Yoshioka, K. Lesion Mimic Mutants. Plant. Signal. Behav. 2008, 3, 764-767. [CrossRef] [PubMed]

5. Qiao, Y.; Jiang, W.; Lee, J.; Park, B.; Choi, M.S.; Piao, R.; Woo, M.O.; Roh, J.H.; Han, L.; Paek, N.C.; et al. SPL28 Encodes a Clathrin-Associated Adaptor Protein Complex 1, Medium Subunit M1 (AP1M1) and Is Responsible for Spotted Leaf and Early Senescence in Rice (Oryza Sativa). New Phytol. 2010, 185, 258-274. [CrossRef]

6. Shirsekar, G.S.; Vega-Sanchez, M.E.; Bordeos, A.; Baraoidan, M.; Swisshelm, A.; Fan, J.; Park, C.H.; Leung, H.; Wang, G.L. Identification and Characterization of Suppressor Mutants of Spl11-Mediated Cell Death in Rice. Mol. Plant Microbe Interact. 2014, 27, 528-536. [CrossRef] [PubMed]

7. Wang, S.; Lei, C.; Wang, J.; Ma, J.; Tang, S.; Wang, C.; Zhao, K.; Tian, P.; Zhang, H.; Qi, C.; et al. SPL33, Encoding an EEF1A-like Protein, Negatively Regulates Cell Death and Defense Responses in Rice. J. Exp. Bot. 2017, 68, 899-913. [CrossRef]

8. Feng, B.H.; Yang, Y.; Shi, Y.F.; Shen, H.C.; Wang, H.M.; Huang, Q.N.; Xu, X.; Lü, X.G.; Wu, J.L. Characterization and Genetic Analysis of a Novel Rice Spotted-Leaf Mutant HM47 with Broad-Spectrum Resistance to Xanthomonas Oryzae Pv. Oryzae. J. Integr. Plant Biol. 2013, 55, 473-483. [CrossRef]

9. Shen, H.C.; Shi, Y.F.; Feng, B.H.; Wang, H.M.; Xu, X.; Huang, Q.N.; Lü, X.G.; Wu, J.L. Identification and Genetic Analysis of a Novel Rice Spotted-Leaf Mutant with Broad-Spectrum Resistance to X Anthomonas Oryzae Pv. Oryzae. J. Integr. Agric. 2014, 13, 713-721. [CrossRef]

10. Chen, Z.; Chen, T.; Sathe, A.P.; He, Y.; Zhang, X.B.; Wu, J.L. Identification of a Novel Semi-Dominant Spotted-Leaf Mutant with Enhanced Resistance to Xanthomonas Oryzae Pv. Oryzae in Rice. Int. J. Mol. Sci. 2018, 19, 3766. [CrossRef]

11. Zhang, F.; Zhuo, D.; Zhang, F.; Huang, L.; Wang, W.; Xu, J.; Cruz, C.V.; Li, Z.; Zhou, Y. Xa39, a Novel Dominant Gene Conferring Broad-Spectrum Resistance to Xanthomonas Oryzae Pv. Oryzae in Rice. Plant Pathol. 2015, 64, 568-575. [CrossRef]

12. Chen, X.; Hao, L.; Pan, J.; Zheng, X.; Jiang, G.; Jin, Y.; Gu, Z.; Qian, Q.; Zhai, W.; Ma, B. SPL5, a Cell Death and Defense-Related Gene, Encodes a Putative Splicing Factor 3b Subunit 3 (SF3b3) in Rice. Mol. Breed. 2012, 30, 939-949. [CrossRef]

13. Ruan, B.; Hua, Z.; Zhao, J.; Zhang, B.; Ren, D.; Liu, C.; Yang, S.; Zhang, A.; Jiang, H.; Yu, H.; et al. OsACL-A2 Negatively Regulates Cell Death and Disease Resistance in Rice. Plant Biotechnol. J. 2019, 17, 1344-1356. [CrossRef]

14. Song, G.; Kwon, C.; Kim, S.; Shim, Y.; Lim, C. The Rice SPOTTED LEAF4 ( SPL4 ) Encodes a Plant Spastin That Inhibits ROS Accumulation in Leaf Development and Functions in Leaf Senescence. Front. Plant Sci. 2019, 9, 1-14. [CrossRef]

15. Sun, C.; Liu, L.; Tang, J.; Lin, A.; Zhang, F.; Fang, J. RLIN1, Encoding a Putative Coproporphyrinogen III Oxidase, Is Involved in Lesion Initiation in Rice. J. Genet. Genom. 2011, 38, 29-37. [CrossRef]

16. Wang, S.; Lim, J.; Kim, S.; Cho, S.; Yoo, S.; Koh, H.; Sakuraba, Y.; Paek, N. Mutation of SPOTTED LEAF3 (SPL3) Impairs Abscisic Acid- Responsive Signalling and Delays Leaf Senescence in Rice. J. Exp. Bot. 2015, 66, 7045-7059. [CrossRef] [PubMed] 
17. Zhou, X.; Liao, H.; Chern, M.; Yin, J.; Chen, Y.; Wang, J.; Zhu, X.; Chen, Z.; Yuan, C.; Zhao, W.; et al. Loss of Function of a Rice TPR-Domain RNA-Binding Protein Confers Broad-Spectrum Disease Resistance. Proc. Natl. Acad. Sci. USA 2018, 115, 3174-3179. [CrossRef]

18. Fekih, R.; Tamiru, M.; Kanzaki, H.; Abe, A. The Rice (Oryza Sativa L.) LESION MIMIC RESEMBLING, Which Encodes an AAA-Type ATPase, Is Implicated in Defense Response. Mol. Genet. Genom. 2015, 290, 611-622. [CrossRef]

19. Sathe, A.P.; Su, X.; Chen, Z.; Chen, T.; Wei, X.; Tang, S.; Zhang, X.; Wu, J.L. Identification and Characterization of a Spotted-Leaf Mutant Spl40 with Enhanced Bacterial Blight Resistance in Rice. Rice 2019, 12, 68. [CrossRef] [PubMed]

20. Mizobuchi, R.; Hirabayashi, H.; Kaji, R.; Nishizawa, Y.; Yoshimura, A.; Satoh, H.; Ogawa, T.; Okamoto, M. Isolation and Characterization of Rice Lesion-Mimic Mutants with Enhanced Resistance to Rice Blast and Bacterial Blight. Plant Sci. 2002, 163, 345-353. [CrossRef]

21. Hammond-kosack, K.E.; Jones, J.D.G. Resistance Gene-Dependent Plant Defense Responses. Plant Cell 1996, 8, $1773-1791$.

22. Meguro, M.; Ito, H.; Takabayashi, A.; Tanaka, R.; Tanaka, A. Identification of the 7-Hydroxymethyl Chlorophyll a Reductase of the Chlorophyll Cycle in Arabidopsis. Plant Cell 2011, 23, 3442-3453. [CrossRef]

23. Sakuraba, Y.; Kim, Y.S.; Yoo, S.C.; Hörtensteiner, S.; Paek, N.C. 7-Hydroxymethyl Chlorophyll a Reductase Functions in Metabolic Channeling of Chlorophyll Breakdown Intermediates during Leaf Senescence. Biochem. Biophys. Res. Commun. 2013, $430,32-37$. [CrossRef]

24. Piao, W.; Han, S.; Sakuraba, Y.; Paek, N. Molecules and Cells Rice 7-Hydroxymethyl Chlorophyll a Reductase Is Involved in the Promotion of Chlorophyll Degradation and Modulates Cell Death Signaling. Mol. Cells 2017, 40, 773-786. [PubMed]

25. Zhao, X.; Jia, T.; Hu, X. HCAR Is a Limitation Factor for Chlorophyll Cycle and Chlorophyll B Degradation in Chlorophyll-BOverproducing Plants. Biomolecules 2020, 10, 1639. [CrossRef] [PubMed]

26. Bansal, R.; Rana, N.; Singh, A.; Dhiman, P.; Mandlik, R.; Sonah, H.; Deshmukh, R.; Sharma, T.R. Evolutionary Understanding of Metacaspase Genes in Cultivated and Wild Oryza Species and Its Role in Disease Resistance Mechanism in Rice. Genes 2020, 11, 1412. [CrossRef]

27. Rani, M.H.; Liu, Q.; Yu, N.; Zhang, Y.; Wang, B.; Cao, Y.; Zhang, Y.; Islam, M.A.; Zegeye, W.A.; Cao, L.; et al. ES5 Is Involved in the Regulation of Phosphatidylserine Synthesis and Impacts on Early Senescence in Rice (Oryza Sativa L.). Plant Mol. Biol. 2020, 102, 501-515. [CrossRef]

28. Mizobuchi, R.; Hirabayashi, H.; Kaji, R.; Nishizawa, Y.; Satoh, H.; Ogawa, T.; Okamoto, M. Differential Expression of Disease Resistance in Rice Lesion-Mimic Mutants. Plant Cell Rep. 2002, 21, 390-396. [CrossRef]

29. Chen, T.; Chen, Z.; Sathe, A.P.; Zhang, Z.H.; Li, L.; Shang, H.H.; Tang, S.; Zhang, X.; Wu, J.L. Characterization of a Novel Gain-of-Function Spotted-Leaf Mutant with Enhanced Disease Resistance in Rice. Rice Sci. 2019, 26, 372-383.

30. Zhang, Y.; Liu, Q.; Zhang, Y.; Chen, Y.; Yu, N.; Cao, Y.; Zhan, X.; Cheng, S.; Cao, L. LMM24 Encodes Receptor-like Cytoplasmic Kinase 109, Which Regulates Cell Death and Defense Responses in Rice. Int. J. Mol. Sci. 2019, 20, 3242. [CrossRef]

31. Li, Y.; Lu, Y.; Shi, Y.; Wu, L.; Xu, Y.; Huang, F.; Guo, X.; Zhang, Y.; Fan, J.; Zhao, J.; et al. Multiple Rice MicroRNAs Are Involved in Immunity against the Blast Fungus Magnaporthe Oryzae. Plant Physiol. 2014, 164, 1077-1092. [CrossRef]

32. Yadav, N.; Sharma, S. Reactive Oxygen Species, Oxidative Stress and ROS Scavenging System in Plants. J. Chem. Pharm. Res. 2016, 8, 595-604.

33. He, Y.; Li, L.; Zhang, Z.; Wu, J.L. Identification and Comparative Analysis of Premature Senescence Leaf Mutants in Rice (Oryza Sativa L.). Int. J. Mol. Sci. 2018, 19, 140. [CrossRef]

34. Lv, X.G.; Shi, Y.F.; Xu, X.; Wei, Y.L.; Wang, H.M.; Zhang, X.B.; Wu, J.L. Oryza Sativa Chloroplast Signal Recognition Particle 43 (OscpSRP43) Is Required for Chloroplast Development and Photosynthesis. PLoS ONE 2015, 10, e0143249. [CrossRef]

35. Zhang, X.; Feng, B.; Wang, H.; Xu, X.; Shi, Y.; He, Y.; Chen, Z.; Sathe, A.P.; Shi, L.; Wu, J. A Substitution Mutation in OsPELOTA Confers Bacterial Blight Resistance by Activating the Salicylic Acid Pathway. J. Integr. Plant Biol. 2018, 60, 160-172. [CrossRef] [PubMed]

36. Tamaoki, D.; Seo, S.; Yamada, S.; Kano, A.; Miyamoto, A.; Shishido, H.; Miyoshi, S.; Taniguchi, S.; Akimitsu, K.; Gomi, K. Jasmonic Acid and Salicylic Acid Activate a Common Defense System in Rice. Plant Signal. Behav. 2013, 8, 8-10. [CrossRef]

37. Al-barzinji, I.M. Spectrophotometric Method Using Different Solvents and SPAD Chlorophyll Meter for Determination Some Photosynthesis Pigments of Bean and Cowpea Plants. Int. J. Eng. Technol. Manag. Appl. Sci. 2015, 3, $108-113$.

38. Huang, L.; Sun, Q.; Qin, F.; Li, C.; Zhao, Y.; Zhou, D.X. Down-Regulation of a SILENT INFORMATION REGULATOR2-Related Histone Deacetylase Gene, OsSRT1, Induces DNA Fragmentation and Cell Death in Rice. Plant Physiol. 2007, 144, 1508-1519. [CrossRef] [PubMed]

39. Ma, X.; Zhang, Q.; Zhu, Q.; Liu, W.; Chen, Y.; Qiu, R.; Wang, B.; Yang, Z.; Li, H.; Lin, Y.; et al. A Robust CRISPR/Cas9 System for Convenient, High-Efficiency Multiplex Genome Editing in Monocot and Dicot Plants. Mol. Plant 2015, 8, 1274-1284. [CrossRef]

40. Toki, S.; Hara, N.; Ono, K.; Onodera, H.; Tagiri, A.; Oka, S.; Tanaka, H. Early Infection of Scutellum Tissue with Agrobacterium Allows High-Speed Transformation of Rice. Plant J. 2006, 47, 969-976. [CrossRef]

41. Kauffman, H.E. An improved technique for evaluating resistance of rice varieties to Xanthomonas Oryzae. Plant Dis. Rep. 1973, 57, 537-541. 